\title{
THE JACOBI SUMS OF ORDER TWENTY-TWO
}

\section{YUN-CHENG ZEE}

AbSTRACT. This paper completes the analysis of the Jacobi sums of order 22 outlined by L. E. Dickson. Furthermore, it is shown that a prime $p$ of the form $22 f+1$ has the binary quadratic decomposition $4 p=u^{2}+11 v^{2}$ and that certain Jacobi sums can be evaluated in terms of $u$ and $v$, where $u$ satisfies $u \equiv 9(\bmod 11)$.

1. Introduction. Let $p$ be a prime of the form $e f+1$ and $g$ a fixed primitive root of $p$. Let $\beta=\exp (2 \pi i / e)$. If $a \equiv g^{j}(\bmod p)$, write ind $a=j$. The Jacobi sum of order $e$ is defined by

$$
R(m, n)=\sum_{a=2}^{p-1} \beta^{m \text { ind } a+n \text { ind (1-a). }}
$$

In 1935, Dickson published three papers [3], [4], [5] on cyclotomy which analyzed Jacobi sums of various orders. Further analyses were later given by Whiteman $(e=10[14], 12[15], 16[13])$, Muskat $(e=15,24,30[9], 14[8])$, Baumert and Fredricksen $(e=9,18[1])$, and the author $(e=13,60[16])$. For $e=20$, see [10].

According to Dickson [4, p. 368, p. 371], $R(m, n)$ is said to be conjugate to $R\left(m^{\prime}, n^{\prime}\right)$ if, for some integer $s$ prime to $e, R\left(m^{\prime}, n^{\prime}\right)$ $= \pm \sigma_{s} R(m, n)$, where $\sigma_{s}$ is the automorphism: $\beta \rightarrow \beta^{s}$. The Jacobi sums can thus be partitioned into conjugate classes whose representatives form a set of reduced Jacobi sums [3, \$16]. For $e=22[4$, p. 373], Dickson chose $R(1, k), k=1,3,5,7,11,13, R(2,2)$ and $R(2,4)$ as the reduced Jacobi sums and stated how $R(1,1), R(1,5), R(1,11)$ and $R(1,13)$ could be obtained linearly from $R(2,2)$ and $R(2,4)$ and that $R(1,7)$ and $R(1,3)$ could be obtained from the equations

$$
\begin{aligned}
& R(1,7)=(-1)^{f} \sigma_{7} R(1,5) \sigma_{5} R(2,4) / \sigma_{13} R(1,5), \\
& R(1,3)=(-1)^{f} R(1,1) R(2,2) / \sigma_{19} R(1,7) .
\end{aligned}
$$

It is the purpose of this paper to derive the linear relations between the reduced Jacobi sums of order 22 outlined by Dickson and to evaluate $R(1,3)$ in terms of a binary quadratic decomposition of $4 p$. The results are drawn in part from the author's doctoral disserta-

Received by the editors February 20, 1970.

AMS 1969 subject classifications. Primary 1041, 1016; Secondary 1066.

Key words and phrases. Cyclotomy, cyclotomic field, Jacobi sum, reduced Jacobi sum, conjugate, cyclotomic number, Gaussian sum, Davenport and Hasse identity, Dickson-Hurwitz sum, binary quadratic decomposition. 
tion under the direction of Professor Muskat at the University of Pittsburgh.

2. Cyclotomy. In this section basic properties concerning the Jacobi sums are gathered for later reference.

A complete set of reduced Jacobi sums of order $e$ may be determined by repeated applications of the following formulas $[3, \S 16]$ :

$$
\begin{aligned}
R(m, n) & =R(n, m)=(-1)^{n f} R(-m-n, n), \\
\sigma_{s} R(m, n) & =R(s m, s n), \quad s \text { prime to } e .
\end{aligned}
$$

Both can be derived from (1).

The Jacobi sum is related to the Gaussian $\operatorname{sum} \tau(n)=\sum_{a=1}^{p-1} \beta^{n \text { ind } a} \zeta^{a}$, where $\zeta=\exp (2 \pi i / p)$, by $[3,(26)]$

$$
R(m, n)=\tau(m) \tau(n) / \tau(m+n),
$$

where none of $m, n$ and $m+n$ is divisible by $e$.

The Gaussian sum satisfies [3, (25)]

$$
\tau(n) \tau(-n)=(-1)^{n f} p
$$

if $e$ does not divide $n$, and $[3,(80)]$

$$
\tau(t) \tau(t+e / 2)=\beta^{-2 t z} \tau(2 t) \tau(e / 2), \quad Z=\text { ind 2, }
$$

if $e$ is even. Equation (6) is a special case of an identity $\left[2,(0.9)_{1}\right]$ established by Davenport and Hasse.

It is immediate from (4) and (5) that if $e$ does not divide $m, n$ or $m+n$, then

$$
R(m, n) R(-m,-n)=p .
$$

The Jacobi sum is important to the determination of the cyclotomic numbers of order $e$, denoted by $(h, k)$. For fixed $h$ and $k,(h, k)$ is the number of solutions $t, z$ of

$$
1+g^{e t+h} \equiv g^{e z+k}(\bmod p), \quad 0 \leqq t, \quad z \leqq f-1 .
$$

It is known $[14,(2.6)]$ that

$$
R(m, n)=(-1)^{m f} \sum_{h, k=0}^{e-1}(h, k) \beta^{m h+n k} .
$$

If $m=n v, R(m, n)$ can be expanded into a finite Fourier series $[14,(2.8)]$ by collecting the exponents of $\beta$ which are congruent modulo $e$ : 


$$
R(v n, n)=(-1)^{v n f} \sum_{a=0}^{e-1} B(a, v) \beta^{n a},
$$

where $B(a, v)=\sum_{h=0}^{e-1}(h, a-v h) . B(a, v)$ is called a Dickson-Hurwitz sum of order $e$ and satisfies [14, (2.12)]

$$
B(a, v)=B(a, e-v-1)
$$

and $[14,(2.13)]$

$$
\begin{aligned}
B(a, 0) & =f-1 & & (a=0), \\
& =f & & (1 \leqq a \leqq e-1) .
\end{aligned}
$$

If $x y=e$ and if $B_{x}(a, v)$ denotes a Dickson-Hurwitz sum of order $x$, then $[9,(61)]$

$$
B_{x}(a, v)=\sum_{b=0}^{y-1} B(a+b x, v) .
$$

3. Linear relations. Let $e=22$. Two special cases of (6) corresponding to $t=1$ and 5 will be used:

$$
\begin{aligned}
& \tau(1) \tau(12)=\beta^{-2 Z} \tau(2) \tau(11), \\
& \tau(5) \tau(16)=\beta^{-10 Z} \tau(10) \tau(11) .
\end{aligned}
$$

Rearranging (12) and using (4), we get

$$
R(1,10)=\beta^{-2 z} R(2,10) \text {. }
$$

By (2) and (3), $R(2,10)=R(10,10)=\sigma_{5} R(2,2)$, so that

$$
R(1,10)=\beta^{-2 Z_{\sigma_{5}}} R(2,2) .
$$

Applying (4) to (12) gives the equation $R(1,1)=\beta^{-22} R(1,11)$. But $R(1,11)=(-1)^{f} R(1,10)$. Hence

$$
R(1,1)=\beta^{-2 Z} R(1,11)=(-1)^{f} \beta^{-4 Z} \sigma_{5} R(2,2) .
$$

By (5), $\tau(6) \tau(16)=(-1)^{f} \tau(11) \tau(11)$. From this equation and (13) we obtain $\tau(1) \tau(5) / \tau(6)=(-1)^{f} \beta^{-10 z} \tau(1) \tau(10) / \tau(11)$. Hence by (4) and (15),

$$
R(1,5)=(-1)^{f} \beta^{10 Z_{\sigma_{5}} R(2,2) .}
$$

$R(1,4) R(1,5)=R(1,1) R(2,4)$ follows from (4). Hence by (16) and (17), $R(1,4)=\beta^{8 z} R(2,4)$. The application of $\sigma_{13}$ to the last equation yields $R(13,8)=\beta^{-6 Z} \sigma_{13} R(2,4)$. Hence by (2), 


$$
R(1,13)=(-1)^{f} \beta^{-6 Z} \sigma_{13} R(2,4) .
$$

We have thus found all the linear relations between the reduced Jacobi sums.

4. Evaluation of $R(1,3)$. Let $q$ be an oddr pimeand let $\theta=\exp (2 \pi i / q)$. It was first proved by Gauss that

$$
\begin{aligned}
\sum_{r=0}^{q-1} \theta^{r} & =\sqrt{ } q & & \text { if } q \equiv 1(\bmod 4), \\
& =i \sqrt{ } q & & \text { if } q \equiv 3(\bmod 4) .
\end{aligned}
$$

Since then several proofs based on different methods have appeared (see [7, pp. 197-218], [6]). The following lemma is an immediate consequence of this result.

Lemma 1. Let $R=\sum \theta^{t}, N=\sum \theta^{s}$, where $t$ (resp. s) runs through the quadratic residues (resp. nonresidues) modulo $q$. Then

$$
\begin{aligned}
R & =(-1+\sqrt{ } q) / 2 & & \text { if } q \equiv 1(\bmod 4), \\
& =(-1+i \sqrt{ } q) / 2 & & \text { if } q \equiv 3(\bmod 4) ; \\
N & =(-1-\sqrt{ } q) / 2 & & \text { if } q \equiv 1(\bmod 4), \\
& =(-1-i \sqrt{ } q) / 2 & & \text { if } q \equiv 3(\bmod 4) .
\end{aligned}
$$

Lemma 2. For $e=22, \beta^{8 z} R(1,3)$ is invariant under the automorphisms $\sigma_{k}, k=1,3,5,9,15$.

Proof. Combining (12) and (13), we get $\tau(2) \tau(5) \tau(16)$ $=\beta^{-8 z} \tau(1) \tau(10) \tau(12)$. By (5), $\tau(10) \tau(12)=\tau(6) \tau(16)$, so that $\tau(2) \tau(5)$ $=\beta^{-8 z} \tau(1) \tau(6)$. Hence

$$
R(2,5)=\beta^{-8 Z} R(1,6)=(-1)^{f} \beta^{-8 z} R(1,15) .
$$

But $\sigma_{9} R(2,5)=R(18,1)=(-1)^{f} R(1,3), \sigma_{9} R(1,15)=R(9,3)=\sigma_{3} R(1,3)$. Hence applying $\sigma_{9}$ to $(19)$, we obtain $\sigma_{3}\left[\beta^{8 Z} R(1,3)\right]=\beta^{8 Z} R(1,3)$. Since $\sigma_{3}^{2}=\sigma_{9}, \sigma_{3}^{3}=\sigma_{5}, \sigma_{3}^{4}=\sigma_{15}$, the proof is complete.

Let $Q$ denote the field of rational numbers. The cyclotomic field $Q(\beta)$ has basis $\left\{1, \beta, \cdots, \beta^{9}\right\}$ with $\beta$ satisfying

$$
\begin{aligned}
\beta^{11}+1 & =0, \\
\sum_{k=0}^{10}(-1)^{k} \beta^{k} & =0 .
\end{aligned}
$$

According to Lemma 2, it is clear that $\beta^{8 z} R(1,3)$ lies in a quadratic extension field over $\boldsymbol{Q}$. 
TheOREM. Let $p$ be a prime of the form $22 f+1$. Then

$$
R(1,3)=(-1)^{f} \beta^{-8} \text { ind } 2(u+i v \sqrt{ } 11) / 2,
$$

where $u$ and $v$ are rational integers satisfying

$$
4 p=u^{2}+11 v^{2}, \quad u \equiv 9(\bmod 11) .
$$

Proof. Let $\delta=(-1)^{f} \beta^{8 z} R(1,3)$. By (8),

$$
\delta=\beta^{8 Z} \sum_{j=0}^{21} B(j, 3) \beta^{j}=\sum_{k=0}^{21} L_{k} \beta^{k},
$$

where $L_{k}=B(k-8 Z, 3), k=0,1, \cdots, 21$. By means of reduction formulas (20) and (21) we obtain

$$
\delta=\sum_{k=0}^{10}\left(L_{k}-L_{k+11}\right) \beta^{k}=\sum_{k=0}^{9} d_{k} \beta^{k},
$$

where

$$
d_{k}=\left(L_{k}-L_{k+11}\right)+(-1)^{k}\left(L_{21}-L_{10}\right), \quad k=0,1, \cdots, 9 .
$$

Applying $\sigma_{8}$ to (22) and simplifying by means of (20) and (21), we get

$$
\begin{aligned}
\sigma_{3} \delta= & \left(d_{0}+d_{7}\right)+\left(-d_{4}-d_{7}\right) \beta+\left(d_{8}+d_{7}\right) \beta^{2}+\left(d_{1}-d_{7}\right) \beta^{3} \\
& +\left(-d_{5}+d_{7}\right) \beta^{4}+\left(d_{9}-d_{7}\right) \beta^{5}+\left(d_{2}+d_{7}\right) \beta^{6} \\
& +\left(-d_{6}-d_{7}\right) \beta^{7}+d_{7} \beta^{8}+\left(d_{3}-d_{7}\right) \beta^{9} .
\end{aligned}
$$

It follows from Lemma 2 that $\sigma_{3} \delta=\delta$. Hence by the uniqueness of representation with respect to the basis of $Q(\beta)$ we can equate the corresponding coefficients in (22) and (24):

$$
\begin{aligned}
& d_{0}=d_{0}+d_{7}, \quad d_{1}=-d_{4}-d_{7}, \quad d_{2}=d_{8}+d_{7}, \quad d_{3}=d_{1}-d_{7}, \quad d_{4}=-d_{5}+d_{7}, \\
& d_{5}=d_{9}-d_{7}, \quad d_{6}=d_{2}+d_{7}, \quad d_{7}=-d_{6}-d_{7}, \quad d_{8}=d_{7}, \quad d_{9}=d_{3}-d_{7} .
\end{aligned}
$$

The ten linear equations yield

$$
d_{2}=d_{6}=d_{7}=d_{8}=0, \quad d_{1}=d_{3}=-d_{4}=d_{5}=d_{9} .
$$

Hence (22) becomes

$\delta=d_{0}+d_{1}\left(\beta+\beta^{3}-\beta^{4}+\beta^{5}+\beta^{9}\right)=d_{0}-d_{1}\left(\theta^{6}+\theta^{7}+\theta^{2}+\theta^{8}+\theta^{10}\right)$, where $\theta=\beta^{2}=\exp (2 \pi i / 11)$. By Lemma 1 ,

$$
\delta=d_{0}+d_{1}(1+i \sqrt{ } 11) / 2=(u+i v \sqrt{ } 11) / 2,
$$

where $u=2 d_{0}+d_{1}, v=d_{1}$. By $(7),|\delta|^{2}=p$, so that $4 p=u^{2}+11 v^{2}$. It remains to show that $u \equiv 9(\bmod 11)$. By $(23)$, 


$$
\begin{aligned}
\sum_{k=0}^{9}(-1)^{k} d_{k}= & \left(L_{0}+L_{2}+\cdots+L_{20}\right) \\
& -\left(L_{1}+L_{3}+\cdots+L_{21}\right)+11 L_{21}-11 L_{10} .
\end{aligned}
$$

On the other hand, by (25), $\sum_{k=0}^{9}(-1)^{k} d_{k}=d_{0}-5 d_{1}$. Hence

$$
\begin{aligned}
d_{0}-5 d_{1} & \equiv\left(L_{0}+L_{2}+\cdots+L_{20}\right)-\left(L_{1}+L_{3}+\cdots+L_{21}\right) \\
& \equiv \sum_{k=0}^{10} B(2 k-8 Z, 3)-\sum_{k=0}^{10} B(2 k+1-8 Z, 3) \\
& \equiv B_{2}(0,1)-B_{2}(1,1)(\bmod 11),
\end{aligned}
$$

by (11). But by (9) and (10), $B_{2}(0,1)=B_{2}(0,0)=11 f-1$. Similarly, $B_{2}(1,1)=B_{2}(1,0)=11 f$. Hence $d_{0}-5 d_{1} \equiv-1(\bmod 11)$. Then $u$ $\equiv 2\left(d_{0}-5 d_{1}\right) \equiv 9(\bmod 11)$. This completes the proof.

The solvability of $4 p=u^{2}+11 v^{2}$ can also be derived from a general theorem in quadratic forms $[12$, p. 273]. The representation of a prime in the form $a x^{2}+b y^{2}, a>0, b>0$, is known to be unique except for the signs of $x$ and $y$. A proof may be found in [11, pp. 190-191]. With a slight modification of the proof it can be shown that $4 p$ $=u^{2}+11 v^{2}$ has an essentially unique solution. In the evaluation of $R(1,3)$ the sign of $u$ is fixed by the congruence $u \equiv 9(\bmod 11)$, whereas that of $v$ depends on the choice of the primitive root $g[3$, pp. 409-410].

\section{REFERENCES}

1. L. D. Baumert and H. Fredricksen, The cyclotomic numbers of order eighteen with applications to difference sets, Math. Comp. 21 (1967), 204-219. MR 36 \#6370.

2. H. Davenport and H. Hasse, Die Nullstellen der Kongruenzzetafunktionen in gewissen zyklischen Fällen, J. Reine Angew. Math. 172 (1934), 151-182.

3. L. E. Dickson, Cyclotomy, higher congruences, and Waring's problem, Amer. J. Math. 57 (1935), 391-424.

4. - Cyclotomy and trinomial congruences, Trans. Amer. Math. Soc. 37 (1935), 363-380.

5. - Cyclotomy when $e$ is composite, Trans. Amer. Math. Soc. 38 (1935), 187-200.

6. T. Estermann, On the sign of the Gaussian sum, J. London Math. Soc. 20 (1945), 66-67. MR 7, 414.

7. E. Landau, Elementary number theory, Teubner, Leipzig, 1927; English transl., Chelsea, New York, 1958. MR 19, 1159.

8. J. B. Muskat, The cyclotomic numbers of order fourteen, Acta Arith. 11 (1966), 263-279. MR 33 \#1302.

9. - On Jacobi sums of certain composite orders, Trans. Amer. Math. Soc. 134 (1968), 483-502. MR 38 \#1075.

10. J. B. Muskat and A. L. Whiteman, The cyclotomic numbers of order twenty, Acta Arith. 17 (1970), 185-216. 
11. T. Nagell, Introduction to number theory, 2nd ed., Chelsea, New York, 1964. MR $30 \# 4714$.

12. H. J. S. Smith, Report on the theory of numbers, Chelsea, New York, 1965.

13. A. L. Whiteman, The cyclotomic numbers of order sixteen, Trans. Amer. Math. Soc. 86 (1957), 401-413. MR 19, 1160.

14. - The cyclotomic numbers of order ten, Proc. Sympos. Appl. Math., vol. 10, Amer. Math. Soc., Providence, R.I., 1960, pp. 95-111. MR 22 \#4682.

15. - The cyclotomic numbers of order twelve, Acta Arith. 6 (1960), 53-76. MR $22 \# 9480$.

16. Y. C. Zee, The Jacobi sums of orders thirteen and sixty and related quadratic decompositions, Math. Z. 115 (1970), 259-272.

California State College, Fullerton, California 92631 